\title{
C2 Model Of Agent-based Radar Network
}

\author{
Zao Zhang \\ National Defence Univ. \\ Beijing, China \\ dazao2001@163.com
}

\author{
Guangya Si \\ National Defence Univ. \\ Beijing, China \\ sgy863@sina.com
}

\begin{abstract}
Radar network is a typical complex adaptive system (CAS). The command and control (C2) of radar network plays a big part in improving the quality of the acquired data and the anti-damage ability of radar network. On the basis of analyzing radar network combat process, a method based on Agent was proposed to establish command and control (C2) model of radar network. The structure of the Agent was put forward and based on that, the adaptive C2 model was represented as a 6-tuple system. The simulation experiment was made in the presumed background and the results showed that the modeling of adaptive command and control of radar network was implemented.
\end{abstract}

Keywords-Agent; radar network command and control; CAS; model; simulation experimentation

\section{INTRODUCTION}

Radar has always played an important role in detecting, classifying and tracking targets of interest in the modern warfare. Along with the development of information technique, radar network has been constructed on the basis of communication network.

Networks and information systems that are being constructed today are complicated. However, radar network is beginning to exhibit traits of a complex adaptive system (CAS) which has the additional important property of being adaptive-i.e., the structure and behavior of the system changes over time in a way which tends to increase its success[1]. Radar network has the ability to adapt to the changing and complex battlefield situations. For example, upon encountering an ARM attack, radar network might carry out some actions, such as stopping radiating. The adaptive behavior of radar network is generated by command and control over the entities in it, and so the adaptive $\mathrm{C} 2$ of radar network plays a big part in improving the quality of the acquired data and the anti-damage ability in combat.

It's an effective way to study radar network through modeling and simulation. Radar network $\mathrm{C} 2$ model can simulate the processes of resource scheduling and the proper action selection of the individual radar according to the rule of its own. In recent years, the works in the field of radar network modeling and simulation focused on analysis and evaluation of radar network operation [2][3], radar network simulation system framework [4] and construction [5][6] of intelligence Surveillance and Reconnaissance(ISR) architecture, etc., which makes the adaptation of radar network insignificant. As radar network possesses the characteristic of the complex adaptive system, the modeling and simulation must exhibit the adaptation. This paper studied on how to model adaptive and dynamic $\mathrm{C} 2$ of radar network through Agent-based modeling and simulation (ABMS). According to ABMS, the Agent-based entity model in complex systems supports the simulation of adaptive behavior. One Agent can receive messages from other Agents and external environment [7], process those messages according to its rules and restrictions and then change its internal status or deliver messages to other Agents. So ABMS is adopted here to model radar network $\mathrm{C} 2$.

\section{STRUCTURE OF AgENT}

This paper takes land-based radar network for air defense as the research object. As shown in Fig.1, Radar network model consists of two kinds of entity Agent---the radar Agent and the fusion center Agent.

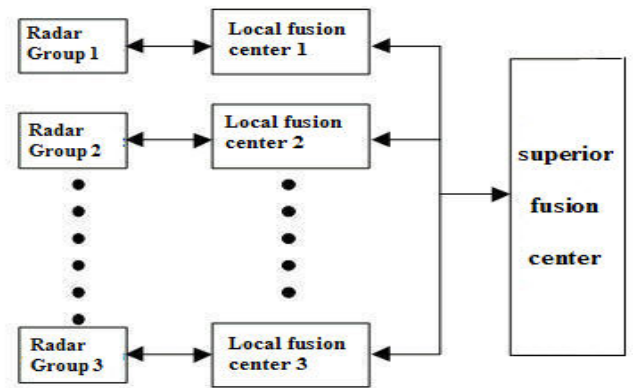

Figure 1. The hierarchy of radar network

The radar Agent has the ability to detect, classify and track targets of interest that may be friend, neutral or enemy. And it takes actions according to its rules and current battlefield situations, or under the command of the local fusion center. The fusion center Agent is capable of keeping aware of battlefield situations. Furthermore, the fusion center Agent also plays a role as commander. It is capable of making decisions according to battlefield situations and sending command and control messages to the subordinate radar Agents. An Agent can share information with others which are networked with it.

To meet the need of modeling radar network $\mathrm{C} 2$, this paper designed the structure [8][9] of the Agent in radar network, shown as figure 2 . The Agent is mainly made up of five parts, which are sensor, message processor, message storage, controller and actor. 


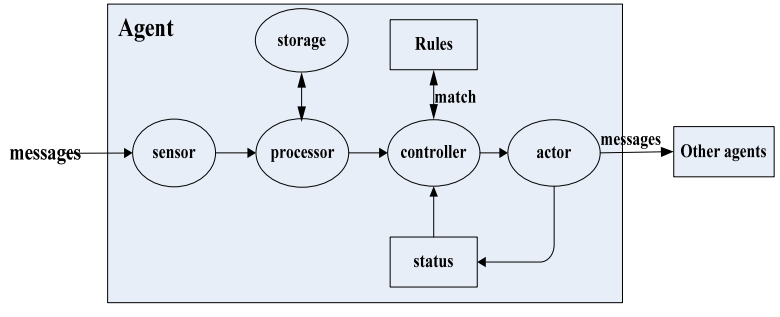

Figure 2. Structure of Agent

The Agent in radar network can receive messages from other Agents and from external environment through the sensor. The message storage stores messages processed by the processor. The message processor can integrate messages from the sensor with the messages stored. The controller gets input from the processor and the status, and determines whether the Agent should execute reactions and which reaction to execute. The actor is responsible for the execution of reaction, and the reaction provides the capability to change status of the Agent or produce messages for communication with other Agents.

\section{DESIGN For RADAR NETWORK C2 MODEL}

Based on the description of structure of the Agent in radar network above, this paper represented the $\mathrm{C} 2$ model as a 6-tuple system:

$\mathrm{M}=<\mathrm{I}, \mathrm{O}, \mathrm{S}, \mathrm{R}, \mathrm{Pt}, \mathrm{Pr}>$;

Where $\mathrm{I}$ is the collection of messages integrated by the processor and taken as input by the controller, $\mathrm{O}$ is the reaction collection. $\mathrm{S}$ is the collection of status of Agent, $\mathrm{R}$ is the rule collection, $\mathrm{Pt}$ is the collection of response priority of messages, and $\mathrm{Pr}$ is the collection of reaction probability. The following will go into details about each parameter.

\section{A. Collection of Integrated Messages - I}

The processor has the ability to integrate input messages from the sensor with messages stored and outputs integrated messages to the controller. When the controller receives specified messages, responses may occur at a given time. The specific message may be a track message, a commanded message, or an alert message, etc.

For example, the radar Agent might be to shut down all emitters if an ARM alert is received and deliver shutdown message to the fusion center Agent. And then the fusion center Agent delivers corresponding commanded message to its subordinate radar Agents and makes them carry out appropriate actions.

\section{B. Status Collection- $S$}

$\mathrm{S}$ include multiple status such as communication condition, pointing mode, operational mode, azimuth and elevation field of view, azimuth and elevation pointing angles, etc. It depends in part on the status of the Agent whether responses can occur. For example, the radar Agent may not be shut down if it is being used to track an engagement.

\section{Reaction Collection - $O$}

Once all conditions for executing a reaction have been met, the reaction can occur at a given time. The reaction collection is as follows:

$\mathrm{O}=\{$ ThreaAlert, RadarCtrl, ProCtrlMsg, AlterCommander $\}$;

ThreaAlert provides the capability to generate threat alert. The radar Agent has the ability to generate various alerts such as ARM alert and Jamming alert. Once the radar Agent has detected jammer emitters or ARM launch, it will forward alert to the fusion center Agent.

RadarCtrl represents radar device control. The radar Agent is given the ability to shut down emitters, control the pointing mode, operational mode and specify the azimuth and elevation field of view, azimuth and elevation pointing angles.

ProCtrlMsg provides the capability to produce commanded messages. For example, after receiving alert messages, the fusion center Agent delivers corresponding control messages to the radar Agent to make them change status.

AlterCommander provides the capability for the Agent to be assigned to a new commander as a response to lost or death of its commander. Once the assignment has been made, the alternate commander can communicate messages with the new subordinate.

\section{Rule Collection $-R$}

$R$ is the rule collection, which is the mapping : $I \times S \rightarrow O$. The rule collection is specified by the model and operates using the collection of integrated messages, the status collection and the reaction collection. Based on an integrated message, a response may occur. The controller obtains the matching rule in terms of the input message and status of the Agent. For example, if $\mathrm{S}=\left\{\mathrm{S}_{1}, \mathrm{~S} 2\right\}, \mathrm{I}=\left\{\mathrm{I}_{1}, \mathrm{I}_{2}\right\}, \mathrm{O}=\left\{\mathrm{O}_{1}, \mathrm{O}_{2}\right\}$, and $\mathrm{R}=\left\{\mathrm{R}_{1}, \mathrm{R}_{2}\right\}, \mathrm{R}_{1}$ :

$\mathrm{I}_{1} \times \mathrm{S}_{2} \rightarrow \mathrm{O}_{1}$ means that when input message is $\mathrm{I}_{1}$ and status is $\mathrm{S}_{2}$, the matching rule is $\mathrm{R}_{1}$ and $\mathrm{R}_{1}$ determines the reaction $\mathrm{O}_{1}$ should occur.

\section{E. Response Priority \& Reaction Probability}

According to the FCFS (First-Come-First-Served) principle, the messages which arrived earlier are picked out to be responded first in the process of simulation. Multiple responses can occur if they don't conflict with a currently executing response, i.e., more than one response is scheduled to control the same radar device. For deconfliction of multiple responses, the model assigned a response priority for each message, with the comparison of which, the controller can pick up the message with the highest priority to respond first. When the response to the message with higher priority is completed, those messages with lower priority will be the next. That's to say, when $n(n \in N \wedge n>1)$ messages which the response to are in conflict have been input to the controller, the collection of those $\mathrm{n}$ messages is $\mathrm{I}^{\prime}\left(\mathrm{I}^{\prime} \subset \mathrm{I}\right) . \exists \mathrm{I}_{\mathrm{i}} \in \mathrm{I}^{\prime}, \mathrm{I}_{\mathrm{i}}(\mathrm{i} \in \mathrm{N})$ has the priority $\mathrm{Pt}_{\mathrm{i}}\left(\mathrm{Pt}_{\mathrm{i}} \in \mathrm{Pt}\right)$. $\forall I_{j} \in I^{\prime} \wedge I_{j} \neq I_{i}(j \in N \wedge j \neq i), I_{j}$ has the priority $\mathrm{Pt}_{\mathrm{j}}\left(\mathrm{Pt}_{\mathrm{j}} \in \mathrm{Pt}\right)$. If $\mathrm{Pt}_{\mathrm{i}}>\mathrm{Pt}_{\mathrm{j}}$, then $\mathrm{I}_{\mathrm{i}}$ should be responded first. 
Assuming that the input to the controller is $\mathrm{I}_{\mathrm{i}}\left(\mathrm{I}_{\mathrm{i}} \in \mathrm{I}\right)$, which is corresponding with the status $S_{i}\left(S_{i} \subset S\right)$. Based on $I_{i}$ and $S_{i}$, the model can get the matching rule $R_{i}\left(R_{i} \in R\right)$, with the help of which, it will choose one or more reactions. These reactions make up the reaction collection $\mathrm{O}_{i}\left(\mathrm{O}_{\mathrm{i}} \subset 0\right)$, and $\mathrm{O}_{\mathrm{i}}$ will be executed. However, in operation, there may exist some $\mathrm{X}$ factors to disturb the execution of some reactions. To fix this problem, the model assigned the probability for each reaction, which is in the range from 0 to 1. Then a random number in the same range will be generated by the actor of the Agent. By comparing the random number and the probability of each reaction, the model can decide whether reactions can be completed. That is, $\forall \mathrm{O}^{\prime}{ }_{\mathrm{k}} \in \mathrm{O}_{\mathrm{i}}, \exists \operatorname{Pr}_{\mathrm{O}_{\mathrm{k}}} \in \mathrm{Pr}$, if $\operatorname{Pr}_{\mathrm{O}_{\mathrm{k}}}>$ Random, then $\mathrm{O}_{\mathrm{k}}^{\prime}$ will be executed by the actor. If $\operatorname{Pr}_{\mathrm{O}^{\prime}} \leq$ Random, then $\mathrm{O}_{\mathrm{k}}^{\prime}$ cannot be executed.

After $\mathrm{O}_{\mathrm{i}}$ has been executed, the Agent responds other messages at the same way. The following is the pseudo-code of the $\mathrm{C} 2$ model:

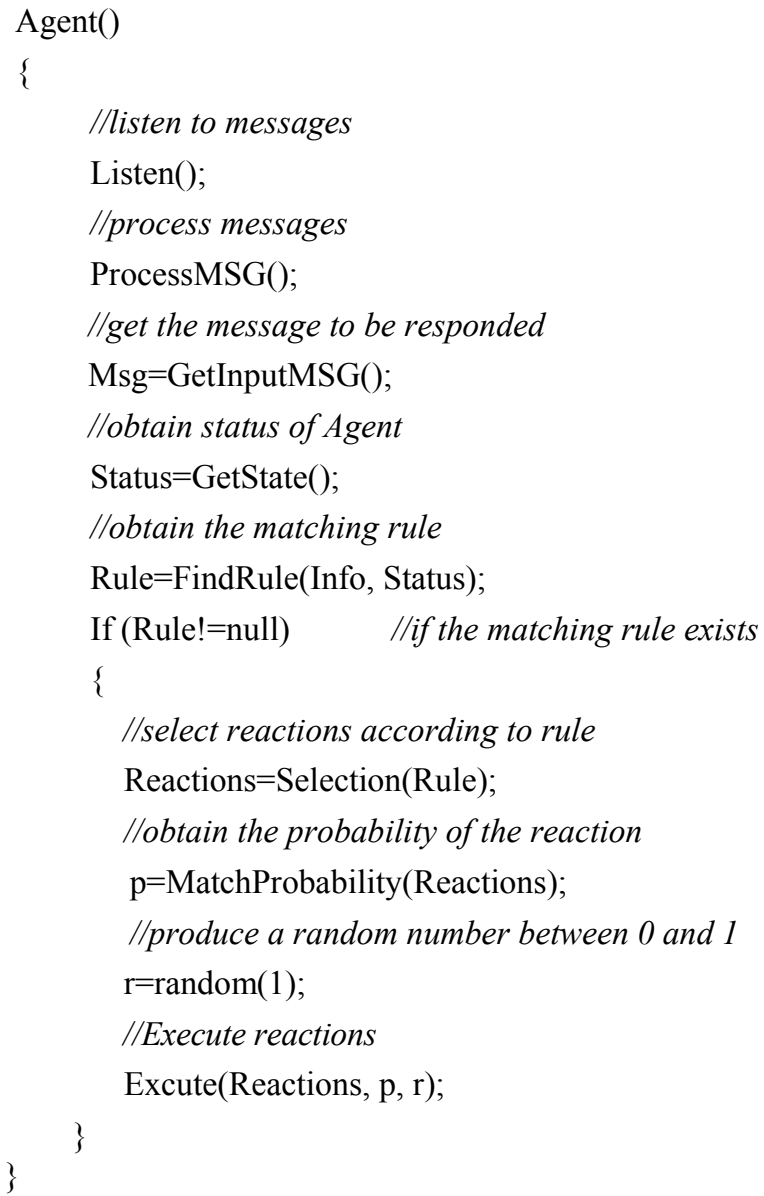

\section{SIMULATION EXPERIMENT}

\section{A. The Scenario}

A typical scenario was used to illustrate the radar network $\mathrm{C} 2$ model proposed above, in which a blue anti-radiation UAV tried to launch air strikes on the radar in the red radar network. The radar network consisted of two radars and a fusion center which was the commander of the two radars. The radar network C2 model was going to be validated with the response to ARM launch.

\section{B. Experimental Result}

\begin{tabular}{|l|l|l|l|}
\hline \multicolumn{1}{|c|}{ Tine } & \multicolumn{1}{|c|}{ Entity } & \multicolumn{1}{c|}{ Event } & \multicolumn{1}{c|}{ Target } \\
\hline 2581.27 & Anti-radiation UAV & Navigating A Cruise & Radar A \\
\hline 2825.05 & Anti-radiation UAT & Gain Radar Signal & Radar A \\
\hline 2843.05 & Radar A & Searching Target & \\
\hline 2843.05 & Radar A & Gain Radar Signal & Anti-radiation UAV \\
\hline 2848.05 & Radar A & ARII Classification & Anti-radiation UAV \\
\hline 2848.05 & Radar A & ARII Alert & Local fusion center \\
\hline 2848.12 & Local fusion center & Sending Control Message & Radar B \\
\hline 2848.12 & Radar A & Shutting Down & \\
\hline 2852.08 & Radar B & Ajusting Antenna Point & \\
\hline 2853.34 & Radar B & FOV Changed & \\
\hline 3183.53 & Anti-radiation UAV & Ending cruising & Radar A \\
\hline 3185.01 & Anti-radiation UAV & Exiting Senario & \\
\hline
\end{tabular}

Figure 3. Experimental result

A simulation experiment was carried out in the implemented simulation system integrated with the radar network $\mathrm{C} 2$ model. The simulation experimental result is presented as Figure 3. An anti-radiation UAV was cruising according to a specified route. At one point, the UAV detected signal emissions from radar $\mathrm{A}$, tracked the signal and tried to launch ARM to engage radar A. Radar A immediately classified the ARM, forwarded ARM alert to the fusion center and shut down its emitters so that the UAV failed to destroy radar A. At the same time, radar B adjusted the antenna point to surveillance area of radar A under command of the local fusion center. The experimental result was in correspondence with reality and the radar network $\mathrm{C} 2$ model was validated.

\section{CONCLUSIONS}

Based on the analysis of radar network combat process, this paper established $\mathrm{C} 2$ model of Agent-based radar network for system-of-systems simulation, validated the model through simulation experimentation, and made a constructive attempt to exhibit the adaptation in the modeling of the complex adaptive system.

\section{ACKNOWLEDGMENT}

This work is supported by National Natural Science Foundation of China under Grant No.61273189.

\section{REFERENCES}

[1] Paul W. Phister Jr. Cyberspace: The Ultimate Complex Adaptive System. THE INTERNATIONAL C2 JOURNAL, vol. 4, no. 2, 2010-2011.

[2] Chen Yon-guang, Li Xiu-he, Shen yang. Networking radar system analysis and evaluation. Beijing: National Defense Industry Press, Jan. 2006.

[3] Jiang Qiu-xi. Introduction to combat networking radar system. Beijing: National Defense Industry Press, Nov. 2010.

[4] LI Xiong, GUO Qi-sheng, WANG Jing-ye. Multi-Agent-based Simulation Model of Multi-sensor for information battlefield. COMPUTER SIMULATION, vol. 23, no. 7, pp. 27-30, 2006.

[5] Lawence A.H.Liang. The Use of Agent Based Simulation for Cooperative Sensing of the Battlefield. Naval Postgraduate School, Dec. 2005 . 
[6] Keith L. Ruegger. ARCHITECTING A NET-CENTRIC OPERATIONS SYSTEM OF SYSTEMS FOR MULTI-DOMAIN AWARENESS. Naval Postgraduate School, Sep. 2008.

[7] HU Xiao-feng, YANG Jing-yu, SI Guang-ya, ZHANG Ming-Zhi. War Complex System Simulation Analysis \& Experimentation[M]. Beijing; NDU Press, Jan. 2008.
[8] ZHANG Lei. The research and design of multilayer distributed intelligent decision support system. Xi'an : Northwest Poly-technic University, 2006.

[9] Yu Xin-an, Mu Xiao-dong, Song Wei. The research and design of Agent in war simulation. Journal of Sichuan Ordnance, vol. 31, no. 11, pp. 134-136, 2010. 